This item was submitted to Loughborough's Research Repository by the author.

Items in Figshare are protected by copyright, with all rights reserved, unless otherwise indicated.

\title{
Maintaining system of systems that are fit for purpose: Effectively and efficiently preparing for the unforeseeable
}

PLEASE CITE THE PUBLISHED VERSION

https://doi.org/10.1109/MSMC.2016.2564099

PUBLISHER

(C) IEEE

VERSION

AM (Accepted Manuscript)

LICENCE

CC BY-NC-ND 4.0

\section{REPOSITORY RECORD}

Hinsley, Steven W., Michael Henshaw, and Carys E. Siemieniuch. 2019. "Maintaining System of Systems That Are Fit for Purpose: Effectively and Efficiently Preparing for the Unforeseeable". figshare.

https://hdl.handle.net/2134/24109. 


\title{
Maintaining System-of-Systems Fit-for-Purpose Effectively and Efficiently Preparing for the Unforeseeable
}

\author{
S.W. Hinsley CEng MIET, Prof. M.J. Henshaw, MIEEE, and Prof. C.E. Siemieniuch. C.ErgHF
}

\begin{abstract}
This paper hypothesizes that a System-of-Systems (SoS) that is not Fit-For-Purpose (FFP) is unfit because it cannot implement the correct, timely and complete transfers of Matter, Energy and Information (MEI) between its constituents and with its external environment that are necessary to achieve a particular result. This research addresses the problem of maintaining a SoS FFP after unpredictable changes in operation, composition or external factors by creating a method, implemented as an engineering process and supported by an analysis technique to enhance the affordance of SoS constituents for MEI transfer (MEIX) and reveal potential undesirable transfers.
\end{abstract}

Index Terms - Affordances, Fit-For-Purpose, System-ofSystems, System Interactions, System Interfaces

\section{INTRODUCTION}

This paper summarizes research addressing the problem of how to keep a System-of-Systems (SoS) Fit-for-Purpose (FFP). The choice of research topic was motivated by the author's observations from working in the defense industry with several companies over a career of 30 plus years. There appeared to be a tendency for delivered products and services that, although meeting their requirements, needed modification to maintain a desired capability from the composing SoS, and hence be made FFP. Changes in the SoS situation, for example the operational environment, requirement or the SoS' capability components often rendered the SoS not FFP due to a combination of the two reasons below in various proportions:

- The SoS capability was degraded and could no longer bring about the desired outcome for which it was designed.

- The SoS needed to provide some different functionality to that for which it was designed in order to achieve the desired outcome.

Causes of FFP loss were dynamic and varied, often due to situational changes dictating that modifications to SoS constituent systems, to recover SoS FFP, had to be made close

Date Submitted: 08/14/2015

This research has been supported by the Loughborough University Graduate School and THALES.

S.W. Hinsley, ESoS Group, School of Electronic, Electrical and Systems Engineering, Loughborough University, Leicestershire, LE11 3TU, UK. (s.w.hinsley2@lboro.ac.uk).

Prof. M.J. Henshaw ESoS Group, School of Electronic, Electrical and Systems Engineering, Loughborough University, Leicestershire, LE11 3TU, UK(m.j.d.henshaw@lboro.ac.uk).

Prof. C.E. Siemieniuch, ESoS Group, School of Electronic, Electrical and Systems Engineering, Loughborough University, Leicestershire, LE11 3TU, UK (c.e.siemieniuch@lboro.ac.uk). to, or at the point of utilization, frequently by the personnel working as part of the SoS using 'work-arounds'. A workaround is an engineering solution that is sufficient but rarely optimal in terms of efficiency or cost. Systems engineering shows that corrective action is most effectively and economically done early in the lifecycle, but it is acknowledged that total avoidance of late-stage modifications is unfeasible, which posed the question "What could be done by suppliers to facilitate maintenance of FFP?" It may be noted that one of the characteristics of a SoS noted by Maier [1] is that it is evolutionary; this implies that development of a SoS always requires the adaptation of an existing (legacy) set of systems: there is no clear sheet.

Section II of this paper discusses the need for SoS fitnessfor-purpose maintenance and the associated personnel, section III makes an assertion of the problem and it's root causes and introduces terminology used by the FFP method described in section IV. Sections V and VI relate FFP to the systems engineering process and outlines how the FFP method is practically employed and exploited. Section VII is an illustration of the application of the FFP process, the enhancement resulting from its use and subsequent exploitation of the enhancement to restore a SoS-level capability lost due to an unforeseen external change. Section VIII draws some conclusions from the work described herein.

\section{RE-CONFIGURABILITY}

\section{A. The Need for Re-configurability}

In his holistic approach to risk management Hopkin [2], notes that risk is a "Circumstance, Action, Situation or Event (CASE) with the ability to impact key dependencies". Such impacts are equivalent to Systems-of-Systems not being FitFor-Purpose (FFP). More generally, the SoS not being FFP (i.e. able to do what the user requires) is often due to unforeseen circumstances, actions, situations or events, so that personnel working as part of the SoS have to modify the constituent systems close to the point of employment so that they can converge towards their aims. If the necessary system modifications are not feasible, the users' subsequent failure to achieve their objectives may have consequences ranging from increased costs to loss of life or property.

CASE that might adversely affect a SoS being FFP can be categorized as "known", "known-unknown", “unknownknown" and "unknown-unknown" [3]. Engineering actions can be taken to enhance fitness, and the better known these CASE are (i.e. the more predictable the CASE) the more 
directly they can be affected by engineering actions. Ring states "A system formalized by prescient design cannot respond to unforeseen situations."[4]. The realization of a systems capability is dependent on the simultaneous readiness of several components, which are described below as eight "Lines of Development" (LoD). The LoDs (described below) are the eight aspects of capability that must be ready simultaneously for the capability in question to be realized. Personnel is one of the eight. Robustness and resilience can be designed-in to a system but any benefit they provide against unknown-unknown factors is largely due to luck. The major LoD contributing to FFP maintenance in this circumstance is often the personnel working as part of the SoS. In support of this, General Sir Rupert Smith states "On every occasion that I have been sent to achieve some military objective in order to serve a political purpose, I, and those with me, have had to change our method and re-organize in order to succeed. Until this was done we could not use our force effectively. On the basis of my lengthy experience, I have come to consider this as normal - a necessary part of every operation" [5].

"Unknown-unknown" CASE poses a significant challenge which needs to be addressed. Building on Ashby's work on "Requisite Variety" [6], Boardman \& Sauser [7] state "The uncertain and unknowable environment in which the SoS must operate presents a mystery of endless proportions, the only proper response to which is to have increasing variety, of a continually emerging nature, to deal with unforeseeable reality that eventually becomes clear and present danger".

This situation means that capability-providing SoS' will have constituent systems that are needed to operate in ways that they were not originally designed to do, will have their service lives extended, and new constituent systems will have to integrate into a brownfield environment. Designers are able to design for agility, robustness and resilience against bounded or foreseeable uncertainty, but attempting to equip against "unknown-unknown" factors appears likely to be inefficient and the design outcome of limited effectiveness. However not to provide anything for personnel to use against unknownunknown factors and leave them to their fate in a world of ever-increasing uncertainty and decreasing resources seems wrong: the motivation for this work is to help them!

\section{B. The 'How, Where and Who' of Maintaining a SoS FFP}

SoS constituent system adaption has the ability to more effectively enhance MEI transfers at low levels than SoS reconfigurability which has been widely utilized. Reconfigurability and adaption are employed together in balance and proportion tailored to each individual case. The FFP method, process and technique facilitates SoS constituent system suppliers to equip their products and services affordably and conveniently with solution components, rather than solutions, to capitalize on the ingenuity and resourcefulness of utilizing personnel to efficiently and effectively address unforeseen changes when they occur. As Dalton commented, "But, ultimately, it is people who turn technology into capability; people who are experts in their profession with a comprehensive knowledge of the operational environment” [8].

\section{SOS CONSTITUENTS, TRANSFERS AND AFFORDANCES}

The UK MoD define Defence LoDs (DLoDs) as "the elements that must be brought together to deliver military capability to operational users" and state that "In addition to the DLoD, Interoperability is included as an overarching theme that must be considered when any DLoD is being addressed" [9]. These elements, commonly known as the components of capability, are:

Training, Equipment, Personnel, Infrastructure, Doctrine and concepts, Organization, Information and Logistics. However, the delivery of capability in a general sense also requires Finance, Legal and Commercial aspects to be ready.

System Engineering has been described as "The management of the emergent properties" [10]. Emergent properties are not attributable to one component of the system, so similarly systems and systems-of-systems engineering has a strong focus on the interactions between constituents, and accordingly this research has a focus on the interactions between the constituents of a SoS. At the fundamental level, these interactions are considered to be transfers of Matter, Energy and Information (MEI). Thus the designed operation of an instantiated SoS of interest is predicated upon the correct, timely and complete transfers of matter, energy and information between the SoS constituent systems to achieve the purpose(s) of the SoS. Designers intentionally design-in mechanisms into SoS constituent systems to make the necessary MEI transfers. This research identifies additional Inherent and Independent Material, Energy and Information (MEI) Sources, Sinks and Bearers (SSBs) in a SoS constituent system that are not managed or captured by its defining documentation. These SSBs may cause undesirable emergent properties when integrated with other SoS constituent systems into a SoS, or may be exploited to enhance the affordance for MEI transfer to address shortfalls.

An illustration of the terms 'Intended', 'Inherent' and 'Independent' used to describe MEI Transfers and SSBs may assist the reader here. For example, a maritime surveillance radar system is the System of Interest (SoI). To supply the electrical radar control cabinet the designer specified an MEI transfer 'affordance' intended to transfer MEI (electrical energy) from the ship's supply to the cabinet by a Steel Wire Armored (SWA) cable routed up from a cable duct beneath the deck.. The inherent mechanical rigidity (mechanical energy Bearer) of the power cable may interfere with the correct operation of the cabinets shock mounts. The structure of the vessel (mechanical energy Bearer) may conduct vibrations from an independent MEI (mechanical energy) source, such as the vessels propulsion engine, to the radar antenna mast (mechanical energy sink) and degrade the radar's stabilization performance.

MEI transfers are considered as affordances, defined at the INCOSE ASEC 2011 conference as "Features that provide the potential for interaction by "Affording the ability to do something, as perceived by the user, to achieve some goal" [11]. 


\section{THE FFP METHOD}

The FFP method is a transform cascade, as shown in Fig. 1 below. The cascade and analyses accommodate SoS constituent systems that themselves are SoS, and also constituent systems collaborative layer design. Although the cascade below suggests a waterfall process, in practice there is feedback, concurrent development and iteration between the transformations.

The method facilitates changes in system capability desired to improve or expand the capabilities of SoS constituent systems to perform system level tasks, as well as those contributing to SoS capability level tasks.

- Transform 1 (Top Left) relates the SoS capabilities in the context of its operational concepts, to the MEI transfers (MEIXs) across its boundary that realize the SoS capabilities.

- Transform 2 in the cascade is a similar transformation to the first transform, but at the SoS constituent system level.

- Transform 3 groups all the prospective SoS constituent system MEIXs into a set of system affordances for MEIX and identifies the major subsystems of interest. This better enables examination and assessment of MEIX enhancement from a subsystem viewpoint.

- Transform 4 analyses the affordances for MEIX and determines a sub-set as candidates for enhancement, by assessment at the system / subsystem level by the relevant specialist discipline engineers.

- Transform 5 associates system design actions with the system MEIX enhancement candidates, guided by the original system design actions and any others that are concurrent with subsystem MEIX enhancement.

The bottom-right 'System PLM Artifacts' represents the Project Lifecycle Management documents, engineering drawings, CAD/CAM models etc. into which the enhancement design actions are integrated.

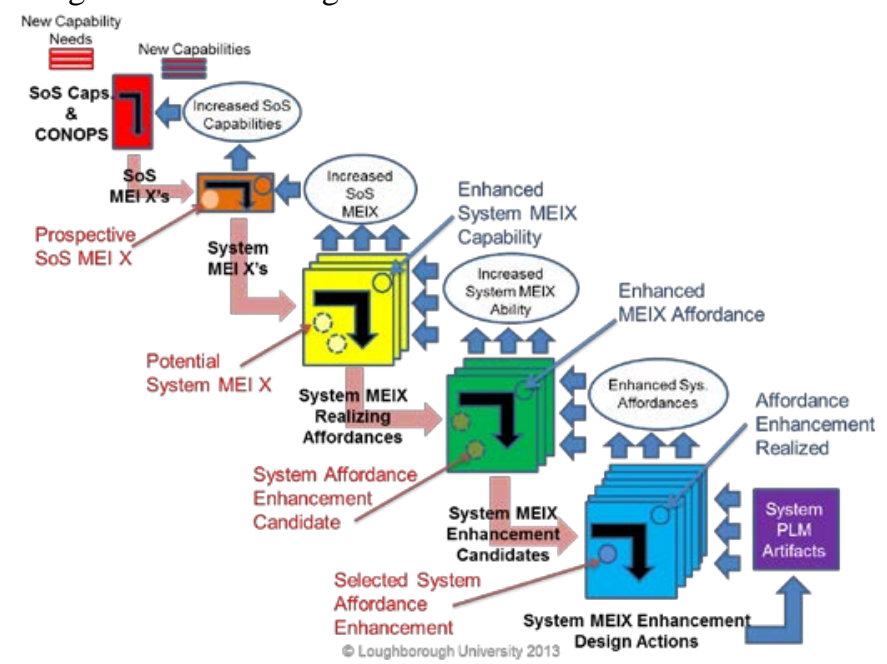

Fig 1. The FFP Method. A cascade of transforms relates top-level SoS capabilities down to constituent systems by the prospective, potential and actual interactions of mass, energy and information.

\section{THE FFP APPLICATION PROCESS}

The FFP process is an instantiation of the FFP method that is tailored to the user organization's particular Product
Lifecycle Management (PLM) system. The FFP process refers to PLM processes and utilizes PLM project artifacts (e.g. user and system requirements, system design and test specifications etc.) familiar to project staff to reduce the opportunity for error and maintain fidelity with the project / System-of-Interest. In systems oriented engineering companies, the product engineering process and the systems engineering process are often one and the same. A common representation of the systems engineering process, used for illustrative purposes only here, is the 'Vee' diagram shown in Fig. 2 [12].

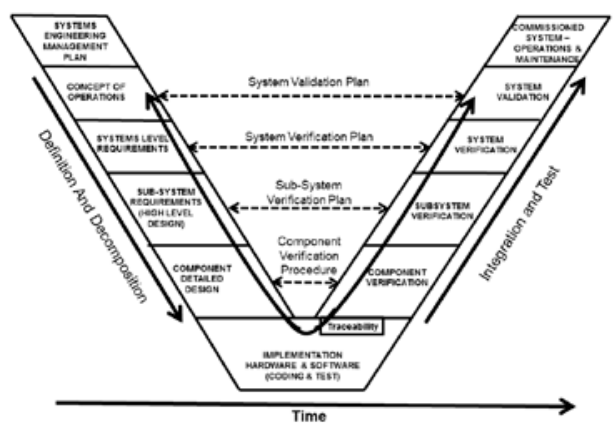

Fig 2. The 'Vee' Diagram. A common representation of the systems engineering process. They range, from conceptual models to assist comprehension of complex systems development to detailed product lifecycle and management models. [12]

The PLM artifacts produced at each stage of the 'Vee' by different company's implementations are functionally similar, but tailored to their individual needs and constraints.

The notion of FFP 'maintenance' is used to emphasize that this process can be applied at any stage shown in Fig. 2 as opportunity arises. The FFP process is not a new approach to design. It offers a new perspective on projects and the engineering process. Analysis used to populate the matrices identifies opportunities to realize affordances at design opportunities such as scheduled major maintenance intervals, obsolescence resolutions, Mid-Life Improvement (MLI) programs and Improvement through Spares programs.

\section{FFP TECHNIQUE AND EXPLOITATION}

To assist designers, a frequency and time domain analysis technique asks the designer to identify the Intended ('designed-for') MEIXs, then examine the MEI SSBs by a 'seismic to light' sweep to show all interaction across the band. For example, the SWA DC power cable conducts AC as well as making a thermal, magnetic and mechanical connection. This technique also contributes to the conventional design process, by identifying undesirable interactions, and what is inherent with design decisions.

The SoI design authority decides what level of provision of MEIX enhancement to implement. For example, enhancement provision in equipment hardware may range from design only, functional models, virtual prototypes, board layout, fitted components to live spares. 


\section{FFP APPLICATION ILLUSTRATION}

\section{A. Deck Approach Light Projector}

To assist aircraft landing on a carrier, a Deck Approach Light Projector (DALP) provides an array of lights on the deck to project beams towards the pilot to indicate the movement of the ship and their aircrafts deviation from the ideal approach angle and landing point. A Wave-Off (WO) lamp in the array illuminates if it is necessary for an approaching aircraft to abort the landing attempt. Fig. 3 below shows a DALP equipment fitted to a carrier.

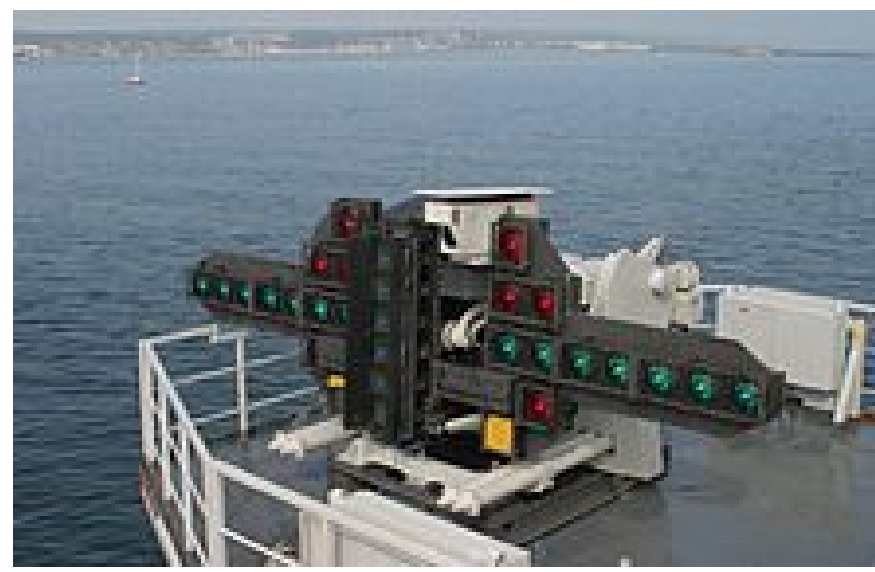

Fig 3. A Deck Approach Light Projector mounted on a naval vessel. [13]

\section{B. DALP Obsolescence Recovery Opportunity}

Obsolescence of some of the original equipment filament bulbs provided an opportunity to reduce downtime and maintenance cost by capitalizing on advances in LED technology. A FFP analysis shows an opportunity to enhance the DALP's ability to transfer MEI in parallel with obsolescence resolution at little extra cost.

The DALP WO MEI transfer affordance is a lamp that when flashed at $1 \mathrm{~Hz}$. instructs the pilot of an approaching aircraft attempting to land that they should abort. The FFP method analysis determined the potential for MEIX enhancement of the DALP's MEI affordances and SSBs, and identifies the potential of the WO affordance for enhanced information transfer.

The on/off and off/on response time of a LED is much faster than that of a conventional incandescent lamp so the faster switching characteristic provides an opportunity to enhance information transfer of the WO affordance by modulating its output. The enhanced WO affordance would provide the carrier an available, non-broadcast, secure, lowlatency and jam-resistant communications link to an approaching aircraft.

The MEI transfer enhancement facilitates a new, secure, unjam-able data transmission from the carrier to an approaching aircraft. The DALP enhancement costs are small, as in this instance the equipment and associated documentation is already scheduled to be modified, and the number of DALPs in-service is small. The enhancement above does not significantly increase component count and type, and does not significantly impact costs from bought-out materials, testing, equipment support publications, training etc. The WO enhancement modification is incorporated into the design actions forming part of the DALP obsolescence recovery project plans and documentation in accordance with the company PLM processes.

\section{DALP MEIX Affordance Enhancement Exploitation}

The carrier is a central part of SoS (a carrier group of vessels) that is conducting military operations in a littoral scenario, whose purpose is to provide Intelligence/Surveillance/Target Acquisition and Reconnaissance (ISTAR) capability to friendly forces ashore. An unforeseen change in the political situation around the carrier groups operations meant that some military tasks being achieved by manned aircraft became untenable. As a consequence of the external change the carrier group was no longer fit for the purpose of providing an ISTAR capability.

Candidate solutions were examined to determine their feasibility, impact and timeliness on both the problem and the capabilities available from the SoS resource, which included the necessary changes and enhancements to the MEI transfers. A preferred solution of Unmanned Air Vehicle (UAV) operations was chosen from a candidate set. Available UAVs have a core capability of operating from land, but do not have the ability to operate from a carrier. The UAV's are normally landed by a human pilot under remote control, but the latency in the control loop is too large to enable the remote pilot to compensate and make adjustments for the movements of the carrier at sea. The enhanced carrier to aircraft information transfer via the light projector is exploited to provide a command link to an unmanned aircraft (UAV) via its panoramic IR/TI camera, auto-tracker and flight control system to provide a low-latency minor control loop to relieve the pilot of compensating for the movements of the vessel, enabling him to apply the flight commands to the UAV landing on the carrier much as he would do for a landing on the ground.

The provision made by the supplier during the DALP obsolescence recovery task was brought on-line whilst the carrier group was on-station, and enabled the available UAVs to be operated from the carrier, thus maintaining fulfilment of the necessary ISTAR tasks without placing pilots, and expensive aircraft containing sensitive intellectual property in harm's way.

\section{CONCLUSION}

The interconnectivity and concomitant complexity of systems is rapidly increasing, meaning that engineers must now think in terms of the fitness of purpose of systems of systems, as opposed to single systems. The FFP method is offered as an holistic-thinking approach that will assist engineers identify concealed MEI sources, sinks, bearers and transfers not otherwise included in SoS/System definition which may lead to unexpected emergent phenomena either problematic and only revealed 'late in the day', or being employed in-service unbeknown and not under the control of the design authority. Examples of such emergence in both 
naval and land-based domains have been found during the test and development of this work. The more complete insight from this approach enhances delivered products / services, and also improves the PLM engineering processes used to create them, and facilitates the task of identifying and implementing SoS adaptation (through reconfiguration at the SoS level or changes to constituent systems) in order to maintain that SoS as fit-for-purpose. This paper has provided an overview of the FFP method and indicated how it may be implemented in organizational processes such that it may offer improved management of a complex SoS, enabling it to be maintained as Fit For Purpose to address new unforeseeable tasks and/or changes, both internal and external, throughout the lifecycle at an affordable scale.

\section{ACKNOWLEDGMENTS}

The author would like to thank the following: M. Sinclair (ESoS Visiting Fellow), A. Johnson (Head of Systems Research, Thales), P. Davies (Former ESoS Visiting Professor), H. Sillitto (Former Systems Engineering Director, Thales), Jean-Luc Garnier (Systems Engineering \& Architecting Director, Thales).

\section{ABbreviations}

\begin{tabular}{|l|l|}
\hline AC & Alternating Current \\
\hline ASEC & Annual Systems Engineering Conference \\
\hline CAD & Computer Aided Design \\
\hline CAM & Computer Aided Manufacture \\
\hline CASE & Circumstance, Action, Situation or Event \\
\hline DC & Direct Current \\
\hline DLoDs & Defense Lines of Development \\
\hline ESoS & Engineering Systems of Systems (Group) \\
\hline FFP & Fit For Purpose \\
\hline INCOSE & International Council on Systems Engineering \\
\hline ISTAR & $\begin{array}{l}\text { Intelligence, Surveillance, Target acquisition and } \\
\text { Reconnaissance }\end{array}$ \\
\hline LoD & Lines of Development \\
\hline MEI & Material/Energy/Information \\
\hline MEIX & MEI transfer \\
\hline MLI & Mid Life Improvement \\
\hline MoD & Ministry of Defense \\
\hline PLM & Project Lifecycle Management \\
\hline SoS & System of Systems \\
\hline SSB & Source/Sink/Bearer \\
\hline SWA & Steel Wire Armored \\
\hline UAV & Unmanned Air Vehicle \\
\hline UK & United Kingdom \\
\hline
\end{tabular}

\section{REFERENCES}

[1] Maier, M.W. (1998), Architecting Principles for Systems-of-Systems, Systems Engineering, Vol. 1, No. 4, pp. 267-284

[2] Hopkin, P., 2002. Holistic Risk Management in Practice 1st ed., London: Witherby \& Co. Ltd. p.3.

[3] Rumsfelt, D. (2002)"Defense.gov News Transcript: DoD News Briefing - Secretary Rumsfeld and Gen. Myers, United States Department of Defense (defense.gov)"
[4] Ring, J, 2012. INCOSE INSIGHT, July 2012 p.42

[5] Smith, R., 2005. The Utility of Force 1st ed., London: Pengiun. p.3.

[6] Ashby, W.R., 1956. An Introduction To Cybernetics, London: Chapman \& Hall Ltd. p.202-208. Available at: http://pespmc1.vub.ac.be/books/IntroCyb.pdf.

[7] Boardman, J. \& Sauser, B., 2006. System of Systems - the meaning of "of." In 2006 IEEE/SMC International Conference on System of Systems Engineering Los Angeles, CA, USA - April 2006. Los Angeles, pp. 118-123.

[8] Dalton, S., 2013. The 21st Century Character of Air Power. , pp.1-11 2013 Sir Sydney Camm Lecture.pdf.

[9] UK MoD. (2015). UK MoD Aquisition System Guidance. Retrieved from https://www.aof.mod.uk/aofcontent/general/sg_dlod.htm

[10] Burrowes, D., \& Squair, M. (1999). managing-the-emergent-propertiesof-design. In A. Limited (Ed.), INCOSE IS (p. 5).

[11] Sillitto, H. (2011). Integrating system thinking system science \& systems engineering. In INCOSE ASEC (p. 20). INCOSE.

[12] Copyright free: authorised under creative commons (https://commons.wikimedia.org/wiki/File:Systems_Engineering_V_dia gram.jpg\#file).

[13] Photo courtesy of www.netmarine.net under creative commons https://commons.wikimedia.org/wiki/File:FS_CdG_Optics.jpg)

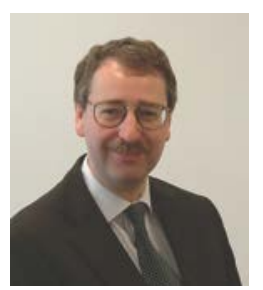

Steve Hinsley is a mature $\mathrm{PhD}$ research student under Professor Mike Henshaw and Professor Carys Siemieniuch in the ESoS Group latterly in the Wolfson School at Loughborough University.

Previously Steve was technical lead of the systems engineering capability for BAE Systems Advanced Technology Centre, and Chief Engineer at the Systems Engineering Innovation Centre. He is a Chartered Engineer, and a member of the IET and INCOSE. He holds a BSc. (Hons.) in Electrical Engineering from De Montfort University Leicester and a MSc. in Advanced Systems Engineering from Loughborough.

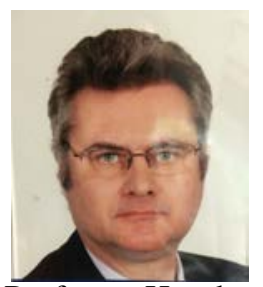

Michael Henshaw is Professor of Systems Engineering and leads the ESoS Research Group. His research focuses on integration and management of complex sociotechnical systems, with a particular emphasis on the challenges of through-life management of systems and capabilities.

Professor Henshaw graduated in applied physics, and his early research focused on laser-plasma interactions, using computational fluid dynamics to investigate various phenomena in applications such as X-ray lasers. He joined British Aerospace (later BAE Systems) as an aerodynamicist and worked for seventeen years in aeronautical engineering tackling problems associated with unsteady aerodynamics (computational and experimental) and, later, multi-disciplinary integration. He was appointed to a chair in Systems Engineering at Loughborough in 2006 to direct the large multi-university, multi-disciplinary program in Network Enabled Capability.

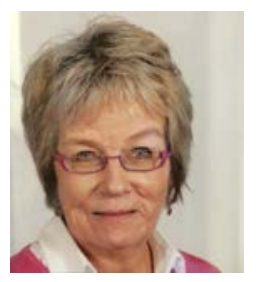

Carys Siemieniuch is a member of the ESoS Research Group. She has both UK professional and European CREE registration as an ergonomist, with expertise across the full range of systemsrelated human factors topics. Her key skills are in knowledge lifecycle 
management systems, organizational and cultural aspects of enterprise modeling techniques, organizational systems architectures, dynamic allocation of function and the design of complex systems. She is active in both the military and civilian domains.

Professor Siemieniuch has extensive and varied consultancy expertise, turning her applied research into practical advice for a number of organizations including the European Parliament, the UK MoD and a range of engineering organizations in the
UK and Europe. She is a reviewer for UK EPSRC and DTI funded programs and a registered 'expert', project reviewer and evaluator for the EU Framework Programs. In addition she is a reviewer for various academic journals and national and international conferences. She has a particular interest in the promotion of women in science, engineering and technology (SET) and is a Mentor on MentorSET: a national mentoring scheme for Women in SET. 